\title{
Foraminal Hematoma Following Lumbar Transforaminal Steroid Injection Causing Reversible Motor Deficit: A Case Report
}

\author{
Mohammed Alyousef $f^{1,2 *}$ and Mikael Orabi ${ }^{1}$ \\ ${ }^{1}$ Department of Neurosurgery, Lariboisière Hospital, France \\ ${ }^{2}$ Division of Neurosurgery, King Abdulaziz University Hospital, Saudi Arabia
}

Submission: April 15, 2017; Published: May 12, 2017

*Corresponding author: Mohammed Alyousef, Department of Neurosurgery, Lariboisière Hospital, 2 Rue Ambroise Paré, 75475 Paris, France, Division of Neurosurgery, King Abdulaziz University Hospital, Jeddah, King Abdulaziz University, Jeddah 22252, Saudi Arabia, Tel: +966-12-640-1000; Fax: +966-12-640 1178; Email: May642_2000@hotmail.com

Abstract

Perineural hematomas are a potentially serious complication of transforaminal epidural steroid injections (TFESI), which are widely used for the treatment of lumbar radicular pain. We present the case of a 70-year-old man with perineural hematoma affecting the L5 nerve root in the lateral recess, resulting in a progressive L5 motor deficit following TFESI. Gadolinium-enhanced Magnetic resonance imaging (MRI) showed a mass consistent with a hematoma occupying the lumbar canal and extending into the right L4-L5 foramen. Surgical evacuation of the hematoma corrected the motor deficit. It is important that physicians are aware of this possible TFESI complication and that closer patient follow-up examinations may be beneficial.

Keywords: Epidural; Perineural hematoma; Foraminal hematoma; Transforaminal epidural injection; Transforaminal

\section{Introduction}

Lumbar radiculopathy is a common disorder with a prevalence of 2.2-8\% [1]. Mechanical compression by a herniated lumbar disc or foraminal stenosis, along with an induced inflammatory response, is thought to underlie radicular pain [2]. Transforaminal epidural steroid injections (TFESI) are widely used for the management of lumbar radicular pain provoked by herniated discopathy and spinal stenosis. The injections deliver glucocorticoids and anesthetics through the intervertebral foramen into the epidural space along the exiting spinal nerves [3]. While the anesthetic component provides immediate pain relief, steroids provide a longer lasting anti-inflammatory effect [4]. As such, the transforaminal route has become popular as a therapeutic intervention which permits the delivery of a higher concentration of steroids with greater specificity, whereas the interlaminar approach has the advantage of providing multilevel spread of the injectate [5]

Even with fluoroscopic guidance, TFESI is not without risks. These include hematoma formation, infection, direct nerve trauma, intravascular injection of medication, subdural injection, disk entry, air embolism, hypersensitivity reactions, and radiation exposure [6]. The incidence and prevalence of epidural hematoma after TFESI is not reported in the literature. However, the approximate incidence after non-traumatic epidural anesthesia, using an interlaminar approach, in a nonheparinized patient is approximately $1 / 200,000$ [7]. In this report, we present a rare case of a hematoma compressing the L4 and L5 nerve roots, resulting in reversible radiculopathy with motor deficit following lumbar TFESI.

\section{Presentation of the case report}

A retired 70-year-old Caucasian man was admitted to our neurosurgery department after developing irreversible radiculopathy with motor deficit following TFESI. Apart from a tachyarrhythmia 3 years prior which was treated with Flecainide $100 \mathrm{mg} / 24 \mathrm{~h}$, the patient was otherwise in good health. Since this was a case report, no ethics committee approval was required. Informed consent was obtained from the patient. The principles of the Declaration of Helsinki were followed.

The patient's symptomatology started in March 2014 as an indolent right-sided lumbogluteal pain, rapidly progressing in severity and radiating to the anterolateral aspect of the right lower limb, down to the dorsum of the right foot. The pain was resistant to medical treatment using Paracetamol, Tramadol and 


\section{Open Access Journal of Neurology \& Neurosurgery}

Lyrica. An MRI of the lower back showed degenerative changes throughout the lumbar spine (L2-L5), an L4-L5 level disc herniation with a cephalad extrusion of disc material compressing the L4 root in the lateral recess, vertebral hemangiomas in L1 and L3, and multiple sacral Tarlov cysts. An EMG performed in September 2014 confirmed a lumbar radiculopathy.

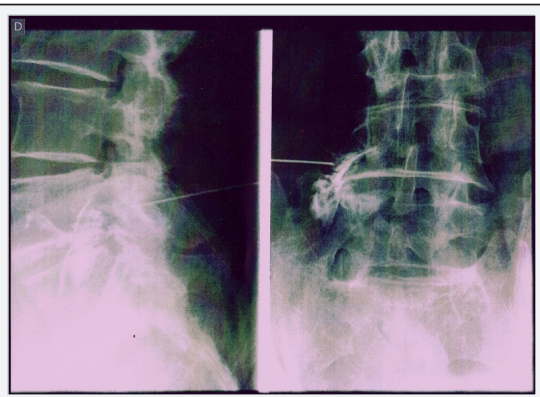

Figure 1: Lateral (a) and anteroposterior (b) fluoroscopy views after injection of contrast medium. The needle is positioned at the exit zone of the right, L4-L5 intervertebral foramen.

After failure of the initial conservative medical treatment, the patient received 3 interlaminar epidural injections between May and September 2014. The pain returned in November 2014, and a right L4-L5 fluoroscopy-guided TFESI was attempted in a private clinic. A $20 \mathrm{G}$ Tuohy needle was used to inject $1.5 \mathrm{ml}$ of the glucocorticoid Altim (Cortivazol) $3.75 \mathrm{mg} / 1.5 \mathrm{ml}$. The contrast agent used was Iopamiron 300 (Iopamidol) (Figure 1). No problems were encountered during the intervention. The two days following the intervention were uneventful and the patient described a reduction in his pain. The third day, however, was marked by progressive reappearance of pain. Furthermore, it was associated with a weakness in the dorsiflexion of the right foot. Thinking it might have been transitional, the patient sought help only four days after the appearance of this deficit.

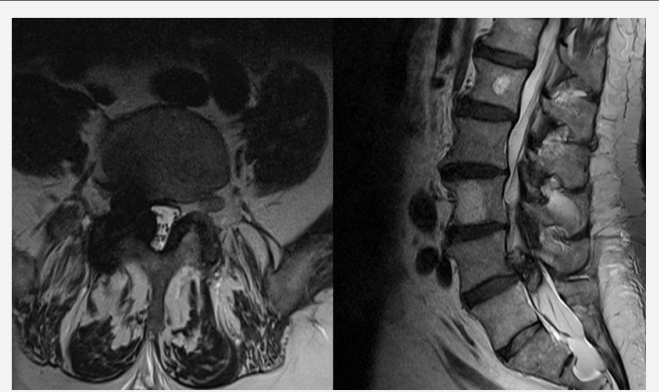

Figure 2: Pre-operative T2-weighted and T1-weighted images showing the right perineural hematoma at the L4-L5 level.

The patient was admitted to our hospital. He was found to have a right foot drop with an inability to walk independently. The motor examination showed a $1 / 5$ motor deficit at the dorsiflexion of his right foot and big toe, an abolition of the right Achilles tendon reflex, and a paresthesia in the dorsum and sole of the foot. Deep sensation was also altered in the right foot. The straight leg raising test showed a right-sided positive Lasègue sign. There was neither sphincter dysfunction nor saddle anesthesia. A subsequent lumbosacral MRI showed a mass measuring $25 \times 15 \mathrm{~mm}$ and occupying the lumbar canal at the L4-L5 level with a right foraminal extension compressing the $\mathrm{L} 4$ nerve root and the L5 nerve root at its emergence. The mass showed a heterogenous hyperintense signal on T1, typical of subacute blood, focal hypointensity on T2-weighted imaging with no enhancement after Gadolinium administration. No changes of the previously known Tarlov cysts had occurred (Figure 2).

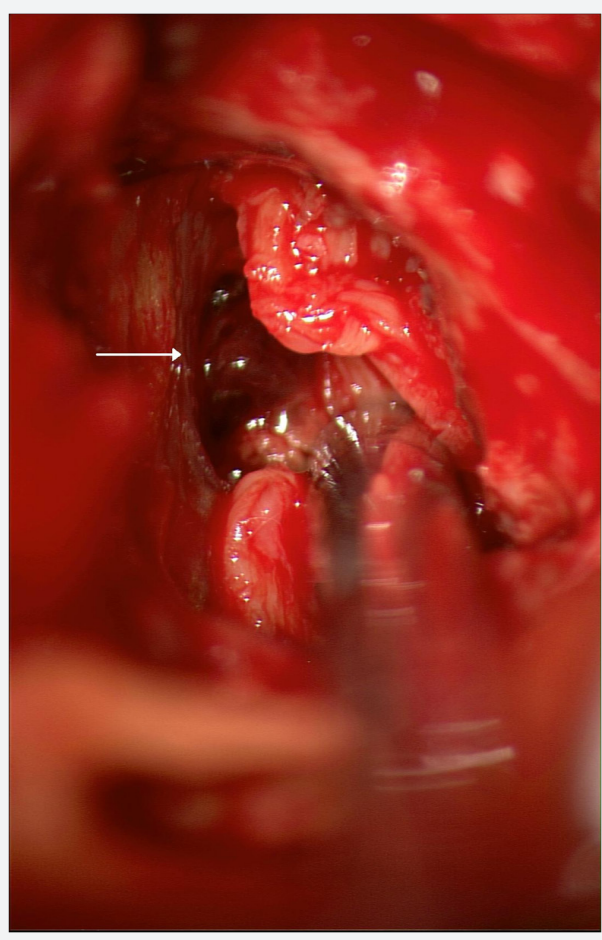

Figure 3: Surgical microscopic view through a right intermyolaminar approach showing the hematoma (white arrow).

The blood analysis revealed no evidence of coagulopathies or bleeding risks, nor did it reveal any inflammation. The patient did not report any bleeding tendencies, nor was he taking any anticoagulants or antiplatelet drugs. His blood analysis showed an international normalized ratio (INR) at 1 , activated partial thromboplastin time at 29 seconds, and a platelet count at $272000 / \mathrm{mm} 3$, which was confirmed by repeated blood analyses during hospitalization. The patient was operated early the following morning. Using an intermyolaminar approach, a right hemilaminectomy was performed and the hematoma was completely evacuated. Blood loss was minimal and no complications were encountered after the surgery (Figure 3). Clinically, the patient showed an improvement in motor deficit with a complete recovery of weakness. However, although improved after surgery, lumbar radicular pain still persists to this day.

\section{Discussion}

In the present case, the patient developed a transforaminal perineural hematoma along the nerve root, discovered four days 
after the appearance of symptoms following TFESI treatment for lumbar radicular pain. The diagnosis was based on a lumbosacral MRI. The patient was not on anticoagulant therapy and did not have a prior history of a bleeding disorder; this is exceedingly rare, as the majority of documented cases are associated with an anticoagulant/antiplatelet therapy or an underlying coagulopathy [7]. Additional identified risk factors for spinal epidural hematoma include anatomic abnormalities of the spinal cord and vertebral column, older age, larger needle size, and needle manipulations after the injection of contrast medium [8]. Radiological assessment using CT-myelography or MRI is helpful to confirm the diagnosis of spinal epidural hematoma. MRI offers the additional advantage of ageing the hematoma through signal characteristics of various stages of hemorrhage. As such, MRI imaging has replaced CT-myelography as the first-choice diagnostic modality for the confirmation of spinal epidural hematoma [9]. In our report, MRI showed a hyperintense signal on T1- and T2-weighted imaging, typical of subacute blood.

Prompt surgical evacuation and decompression has been a standard treatment of spinal epidural hematoma presenting with neurologic deficit to prevent further deterioration. The neurologic prognosis after a surgical decompression depends on the severity of preoperative neurologic deficits, time interval from symptom onset to surgery and the duration of maximum deficit. In a case series by Lawton et al. [10] surgical decompression of spinal epidural hematoma has a mortality of around 3\% and a neurological morbidity of $0 \%$, with neurological improvement expected in $87 \%$ and complete recovery observed in $43 \%$ of patients.

\section{Conclusion}

In conclusion, spinal epidural hematoma is a rare complication of epidural steroid injections that can develop even in patients without any obvious risk factors. We emphasize the importance of a clinical evaluation at a regular interval after epidural injection. In addition, patients should be made aware of the early signs of spinal cord, radicular, and cauda equina compression, such as severe back pain, motor or sensory deficits, and sphincteric dysfunction. Prompt referral to a spine surgeon for an eventual surgical decompression might avert a catastrophic consequence to a minimally invasive pain procedure.

\section{References}

1. Frontera WR, Silver JK, Rizzo TD (2008) Essentials of Physical Medicine and Rehabilitation: Musculoskeletal Disorders, Pain, and Rehabilitation. ( $2^{\text {nd }}$ edn) PA: Saunders/Elsevier, Philadelphia, USA, pp. $1-952$.

2. Saal JS (1995) The role of inflammation in lumbar pain. Spine 20(16): 1821-1827.

3. Desai MJ, Dua S (2014) Perineural hematoma following lumbar transforaminal steroid injection causing acute-on-chronic lumbar radiculopathy: A case report. Pain Pract 14(3): 271-277.

4. Hession WG, Stanczak JD, Davis KW, Choi JJ (2004) Epidural steroid injections. Semin Roentgenol 39(1): 7-23.

5. Desai MJ, Shah B, Sayal PK (2011) Epidural contrast flow patterns of transforaminal epidural steroid injections stratified by commonly used final needle-tip position. Pain Med 12(6): 864-870.

6. Goodman BS, Posecion LW, Mallempati S, Bayazitoglu M (2008) Complications and pitfalls of lumbar interlaminar and transforaminal epidural injections. Curr Rev Musculoskelet Med 1(3-4): 212-222.

7. Horlocker TT, Wedel DJ, Rowlingson JC, Enneking FK, Kopp SL, et al. (2010) Regional anesthesia in the patient receiving antithrombotic or thrombolytic therapy: American society of regional anesthesia and pain medicine evidence-based guidelines (third edition). Reg Anesth Pain Med 35(1): 64-101.

8. Tiso RL, Cutler T, Catania JA, Whalen K (2004) Adverse central nervous system sequelae after selective transforaminal block: The role of corticosteroids. Spine J 4(4): 468-474.

9. Rothfus WE, Chedid MK, Deeb ZL, Abla AA, Maroon JC, et al. (1987) MR imaging in the diagnosis of spontaneous spinal epidural hematomas. J Comput Assist Tomogr 11(5): 851-854.

10. Lawton MT, Porter RW, Heiserman JE, Jacobowitz R, Sonntag VK, et al. (1995) Surgical management of spinal epidural hematoma: Relationship between surgical timing and neurological outcome. J Neurosurg 83(1): 1-7.

\section{Your next submission with Juniper Publishers will reach you the below assets}

- Quality Editorial service

- Swift Peer Review

- Reprints availability

- E-prints Service

- Manuscript Podcast for convenient understanding

- Global attainment for your research

- Manuscript accessibility in different formats

( Pdf, E-pub, Full Text, Audio)

- Unceasing customer service

Track the below URL for one-step submission https://juniperpublishers.com/online-submission.php 